\title{
Analytic Method for Solution the Heat Equation
}

\author{
Iman. I. Gorial
}

Department of Mathematics, College of Education for Pure Science / Ibn Al-Haitham, University Baghdad Iraq

Abstract: In this paper an efficient modification of Adomian decomposition method is introduced for solving heat equation. Tested for some examples and the obtained results demonstrate efficiency of the proposed method. The results were presented in tables and figure using the MathCAD 12 and Matlab software package.

Key words: Modified decomposition method, Heat equation, Nonlocal problem.

\section{Introduction}

Adomian decomposition method can solve large classes of linear and nonlinear differential equations and it is much simpler in computation and quicker in convergence than any other method available in the open literature [1,2]. A variety of modifications to Adomian decomposition method have been reported. Wazwaz presented a strong modification of ADM that accelerates the rapid convergence of the series solution [3, 4]. E. Babolian et al. introduced the restart method to solve the equation $\mathrm{f}(\mathrm{x})=0$ [5], and the integral equations [6]. H. Jafari et.al used a correction of decomposition method for ordinary and nonlinear systems of equations and show that the correction accelerates the convergence $[7,8]$.

In this paper, we present computationally efficient numerical method for solving the heat equation:

$$
\begin{aligned}
& D_{t} u(x, t)=D_{x x} u(x, t)+q(x, t) \\
& u(x, 0)=f(x), 0 \leq x \leq 1 \\
& u(0, t)=\int_{0}^{1} \phi(x, t) u(x, t) d x+g_{1}(t), 0<t \leq T \\
& u(1, t)=\int_{0}^{1} \psi(x, t) u(x, t) d x+g_{2}(t), 0<t \leq T
\end{aligned}
$$

Where $f, g_{1}, g_{2}, \phi, \psi$ and $\mathrm{q}$ are known functions, $\mathrm{T}$ is given constant.

\section{Solution Heat Equation by Modified Adomian's Decomposition Method}

In this section, we will discuss the use of the MDM for the solution of heat equation with nonlocal boundary conditions given in (1). In this method we assume that

$$
u(x, t)=\sum_{n=0}^{\infty} u_{n}(x, t)
$$

Can be rewritten Equation (1):

$$
L_{t} u(x, t)=L_{x x} u(x, t)+q(x, t)
$$

Where

$$
L_{t}(\cdot)=\frac{\partial}{\partial t}(\cdot)
$$

and

$$
L_{x x}=\frac{\partial^{2}}{\partial x^{2}},
$$

The inverse $L^{-1}$ is assumed an integral operator given by 


$$
L^{-1}=\int_{0}^{t}(\cdot) d t
$$

Take the operator $L^{-1}$ on both sides of Equation (5) we have

$$
L^{-1}\left(L_{t} u((x, t))\right)=L^{-1}\left(L_{x x}(u(x, t))\right)+L^{-1}(q(x, t))
$$

Therefore, we can write,

$$
u(x, t)=u(x, 0)+L_{t}^{-1}\left(L_{x x}\left(\sum_{n=0}^{\infty} u_{n}\right)\right)+L^{-1}(q(x, t))
$$

The modified decomposition method was introduced by Wazwaz [11]. This method is based on the assumption that the function $H(x)$ can be divided into two parts, namely $H_{1}(x)$ and $H_{2}(x)$. Under this assumption we set

$$
H(x)=H_{1}(x)+H_{2}(x)
$$

Then the modification

$$
\begin{aligned}
& u_{0}=H_{1} \\
& u_{1}=H_{2}+L_{t}^{-1}\left(L_{x x} u_{0}\right) \\
& u_{n+1}=L_{t}^{-1}\left(L_{x x}\left(\sum_{n=0}^{\infty} u_{n}\right)\right), \mathrm{n}>1
\end{aligned}
$$

\section{Numerical Illustration}

In this paper, we will apply the numerical method to solve heat equation.

\section{Example 1:}

Consider heat equation with nonlocal boundary conditions for the equation (1), as taken in [9]:

$$
\begin{aligned}
& D_{t} u(x, t)=D_{x}^{2} u(x, t)+\frac{-2\left(x^{2}+t+1\right)}{(t+1)^{3}} \\
& u(x, 0)=x^{2}, 0 \leq x \leq 1 \\
& u(0, t)=\int_{0}^{1} x u(x, t) d x+\frac{1}{4(t+1)^{2}}, 0<t \leq 1 \\
& u(1, t)=\int_{0}^{1} x u(x, t) d x+\frac{3}{4(t+1)^{2}}, 0<t \leq 1
\end{aligned}
$$

We apply the above proposed method; we obtain:

$$
\begin{aligned}
& u_{0}(x, t)=\left(\frac{x}{t+1}\right)^{2} \\
& u_{1}(x, t)=0 \\
& u_{2}(x, t)=0 \\
& u_{3}(x, t)=0
\end{aligned}
$$

Then the series form is given by:

$$
u(x, t)=u_{0}(x, t)+u_{1}(x, t)+u_{2}(x, t)+u_{3}(x, t)
$$




$$
=\left(\frac{x}{t+1}\right)^{2}
$$

This is the exact solution $u(x, t)=\left(\frac{x}{t+1}\right)^{2}$

Table 1 shows some of the analytical solutions for heat equation obtained for different values and comparison between exact solution and analytical solution, the plot of the exact solution surface is shown in Figure 1 and Figure 2 is shown the numerical solution surface for heat equation.

Table1. Some of comparison between exact solution and analytical solution

\section{For example 1}

\begin{tabular}{|l|l|l|l|l|}
\hline $\mathbf{x}$ & $\mathbf{t}$ & Exact Solution & Modified Adomian Decomposition Method & $\left|\mathrm{u}_{\mathrm{ex}}-\mathrm{u}_{\mathrm{MADM}}\right|$ \\
\hline 0 & 1 & 0.0000 & 0.0000 & 0.0000 \\
\hline 0.1 & 1 & 0.0025 & 0.0025 & 0.0000 \\
\hline 0.2 & 1 & 0.0100 & 0.0100 & 0.0000 \\
\hline 0.3 & 1 & 0.0230 & 0.0230 & 0.0000 \\
\hline 0.4 & 1 & 0.0400 & 0.0400 & 0.0000 \\
\hline 0.5 & 1 & 0.0630 & 0.0630 & 0.0000 \\
\hline 0.6 & 1 & 0.0900 & 0.0900 & 0.0000 \\
\hline 0.7 & 1 & 0.1230 & 0.1230 & 0.0000 \\
\hline 0.8 & 1 & 0.1600 & 0.1600 & 0.0000 \\
\hline 0.9 & 1 & 0.2030 & 0.2030 & 0.0000 \\
\hline 1 & 1 & 0.2500 & 0.2500 & 0.0000 \\
\hline
\end{tabular}

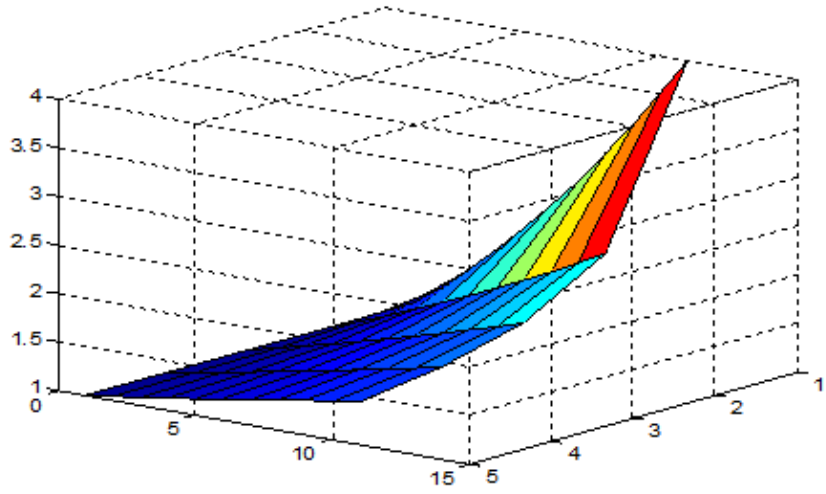

Fig. 1: Exact solution

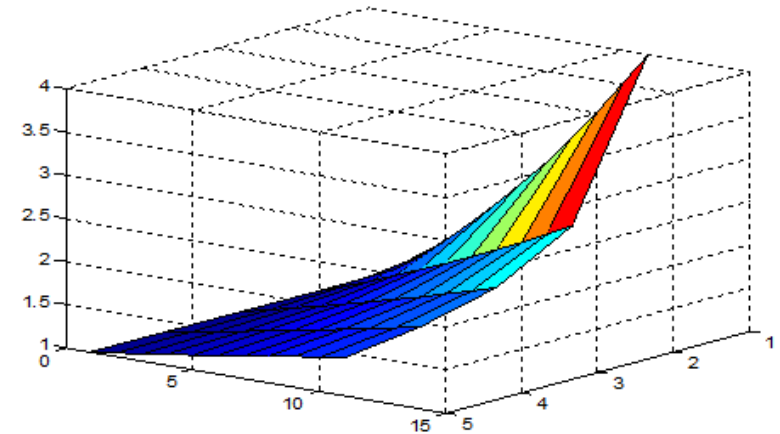

Fig. 2: Numerical solution

\section{Example 2:}

Consider the problem (1) with the following conditions, as taken in [9]:

$D_{t} u(x, t)=D_{x}^{2} u(x, t)$

$u(x, 0)=0.5 x^{2}, 0 \leq x \leq 1$ 
$u(1, t)=1,0<t<T$

$\int_{0}^{b} u(x, t) d x=m(t)=0.75 t+\frac{1}{6}(0.75)^{3}$

where $\mathrm{b} \in[0,1]$.

Now after modified decomposition method, we obtain:

$u_{0}(x, t)=0.5 x^{2}+t$

$u_{1}(x, t)=0$

$u_{2}(x, t)=0$

$u_{3}(x, t)=0$

Then the series form is given by:

$u(x, t)=u_{0}(x, t)+u_{1}(x, t)+u_{2}(x, t)+u_{3}(x, t)$

$=0.5 x^{2}+t$

Which gives the exact solution $u(x, t)=0.5 x^{2}+t$.

Table 2 shows part the analytical solutions for heat equation obtained for different values and comparison between exact solution and analytical solution. Figure 3 and Figure 4 show the plot of the exact and the numerical solution surface for heat equation respectivel

Table2. Some of comparison between exact solution and analytical solution

For example 2 when $t=1,2$

\begin{tabular}{|l|l|l|l|l|}
\hline $\mathbf{X}$ & $\mathbf{t}$ & Exact Solution & Modified Adomian Decomposition Method & $\left|\mathrm{u}_{\mathrm{ex}}-\mathrm{u}_{\text {MADM }}\right|$ \\
\hline 0 & 1 & 1.020 & 1.020 & 0.000 \\
\hline 0.1 & 1 & 1.005 & 1.005 & 0.000 \\
\hline 0.2 & 1 & 1.020 & 1.020 & 0.000 \\
\hline 0.3 & 1 & 1.045 & 1.045 & 0.000 \\
\hline 0.4 & 1 & 1.080 & 1.080 & 0.000 \\
\hline 0.5 & 1 & 1.125 & 1.125 & 0.000 \\
\hline 0.6 & 1 & 1.180 & 1.180 & 0.000 \\
\hline 0.7 & 1 & 1.245 & 1.245 & 0.000 \\
\hline 0.8 & 1 & 1.320 & 1.320 & 0.000 \\
\hline 0.9 & 1 & 1.405 & 1.405 & 0.000 \\
\hline 1 & 1 & 1.500 & 1.500 & 0.000 \\
\hline 0 & 2 & 0.000 & 0.000 & 0.000 \\
\hline 0.1 & 2 & 2.005 & 2.005 & 0.000 \\
\hline 0.2 & 2 & 2.020 & 2.020 & 0.000 \\
\hline 0.3 & 2 & 2.045 & 2.045 & 0.000 \\
\hline 0.4 & 2 & 2.080 & 2.080 & 0.000 \\
\hline 0.5 & 2 & 2.125 & 2.125 & 0.000 \\
\hline 0.6 & 2 & 2.180 & 2.180 & 0.000 \\
\hline 0.7 & 2 & 2.245 & 2.245 & 0.000 \\
\hline 0.8 & 2 & 2.320 & 2.320 & 0.000 \\
\hline 0.9 & 2 & 2.405 & 2.405 & 0.000 \\
\hline 1 & 2 & 2.500 & 2.500 & 0.000 \\
\hline
\end{tabular}

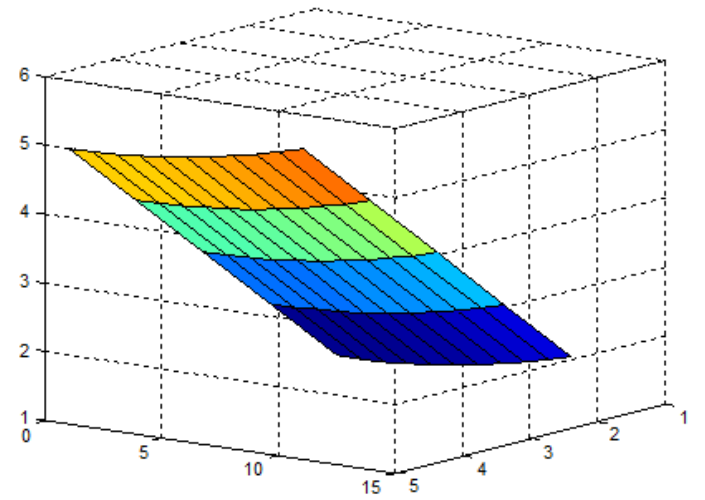

Fig. 3: Exact solution 


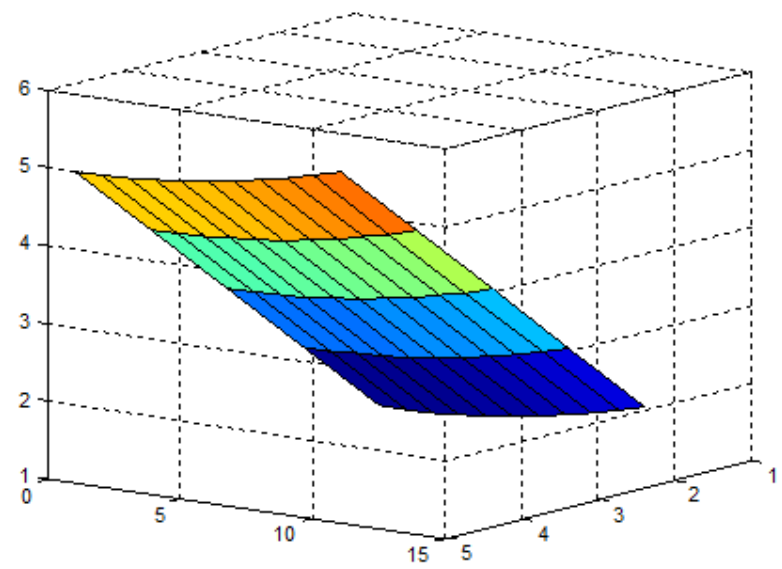

Fig. 4: Numerical solution

\section{Example 3:}

Consider the problem (1) with the following boundary and initial conditions, as taken in [9]

$D_{t} u(x, t)=D_{x}^{2} u(x, t)-30 x^{4}+6 t^{5}$

$u(x, 0)=x^{6}, 0 \leq x \leq 1$

$u(0, t)=\int_{0}^{1} 0.2 u(x, t) d x+\frac{4}{5} t^{6}-\frac{1}{35}, 0<t \leq T$

$u(1, t)=\int_{0}^{1} 0.4 u(x, t) d x+\frac{3}{5} t^{6}+\frac{33}{35}, 0<t \leq T$

Now we apply the above modified decomposition method, we obtain:

$u_{0}(x, t)=x^{6}+t^{6}$

$u_{1}(x, t)=0$

$u_{2}(x, t)=0$

$u_{3}(x, t)=0$

Then the series form is given by:

$$
\begin{aligned}
u(x, t) & =u_{0}(x, t)+u_{1}(x, t)+u_{2}(x, t)+u_{3}(x, t) \\
& =x^{6}+t^{6}
\end{aligned}
$$

This is the exact solution $u(x, t)=x^{6}+t^{6}$.

Table 3 shows some the analytical solutions for heat equation obtained for different values and comparison between exact solution and analytical solution. Figure 5 and Figure 6 show the plot of the exact solution surface and the numerical solution surface for heat equation respectively.

Table3. Some of comparison between exact solution and analytical solution

For example 3 when $\mathrm{t}=1,2,3$

\begin{tabular}{|l|l|l|l|l|}
\hline $\mathbf{x}$ & $\mathbf{t}$ & Exact Solution & Modified Adomian Decomposition Method & $\mid \mathrm{u}_{\mathrm{ex}}-\mathrm{u}_{\mathrm{MADM}}$ \\
\hline 0 & 1 & 0.00000 & 0.00000 & 0.0000 \\
\hline 0.1 & 1 & 1.00000 & 1.00000 & 0.0000 \\
\hline 0.2 & 1 & 1.00000 & 1.00000 & 0.0000 \\
\hline 0.3 & 1 & 1.00100 & 1.00100 & 0.0000 \\
\hline 0.4 & 1 & 1.00400 & 1.00400 & 0.0000 \\
\hline 0.5 & 1 & 1.01600 & 1.01600 & 0.0000 \\
\hline 0.6 & 1 & 1.04700 & 1.04700 & 0.0000 \\
\hline 0.7 & 1 & 1.11800 & 1.11800 & 0.0000 \\
\hline 0.8 & 1 & 1.26200 & 1.26200 & 0.0000 \\
\hline 0.9 & 1 & 1.53100 & 1.53100 & 0.0000 \\
\hline 1 & 1 & 2.00000 & 2.00000 & 0.0000 \\
\hline 0 & 2 & 0.00000 & 0.00000 & 0.0000 \\
\hline
\end{tabular}




\begin{tabular}{|c|c|c|c|c|}
\hline 0.1 & 2 & 64.0000 & 64.0000 & 0.0000 \\
\hline 0.2 & 2 & 64.0000 & 64.0000 & 0.0000 \\
\hline 0.3 & 2 & 64.0010 & 64.0010 & 0.0000 \\
\hline 0.4 & 2 & 64.0040 & 64.0040 & 0.0000 \\
\hline 0.5 & 2 & 64.0160 & 64.0160 & 0.0000 \\
\hline 0.6 & 2 & 64.0470 & 64.0470 & 0.0000 \\
\hline 0.7 & 2 & 64.1180 & 64.1180 & 0.0000 \\
\hline 0.8 & 2 & 64.2620 & 64.2620 & 0.0000 \\
\hline 0.9 & 2 & 64.5310 & 64.5310 & 0.0000 \\
\hline 1 & 3 & 65.0000 & 65.0000 & 0.0000 \\
\hline 0 & 3 & 0.00000 & 0.00000 & 0.0000 \\
\hline 0.1 & 3 & 729.000 & 729.000 & 0.0000 \\
\hline 0.2 & 3 & 729.000 & 729.000 & 0.0000 \\
\hline 0.3 & 3 & 729.001 & 729.001 & 0.0000 \\
\hline 0.4 & 3 & 729.004 & 729.004 & 0.0000 \\
\hline 0.5 & 3 & 729.016 & 729.016 & 0.0000 \\
\hline 0.6 & 3 & 729.047 & 729.047 & 0.0000 \\
\hline 0.7 & 3 & 729.118 & 729.118 & 0.0000 \\
\hline 0.8 & 3 & 729.262 & 729.262 & 0.0000 \\
\hline 0.9 & 3 & 729.531 & 729.531 & 0.0000 \\
\hline 1 & 3 & 730.000 & 730.000 & 0.0000 \\
\hline
\end{tabular}

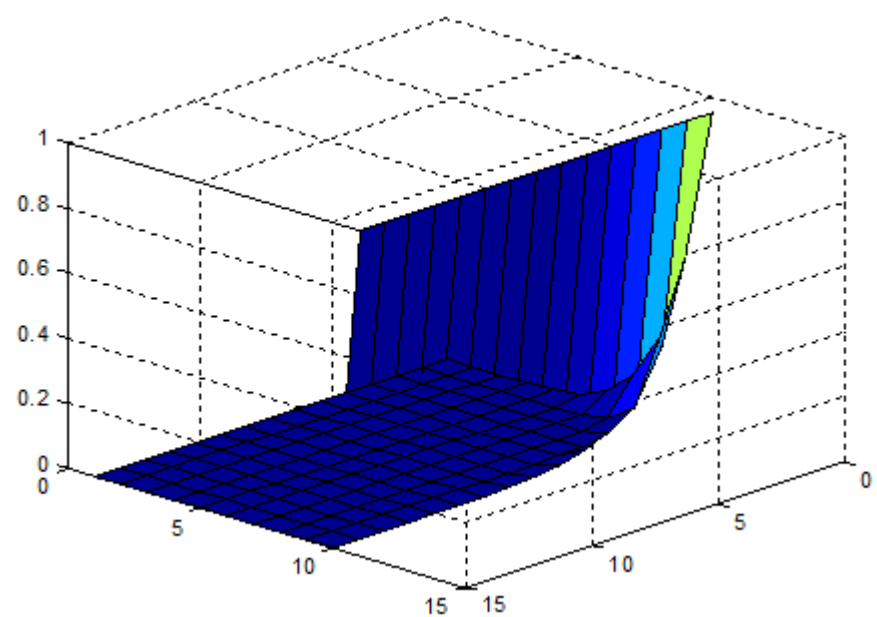

Fig. 5: Exact solution

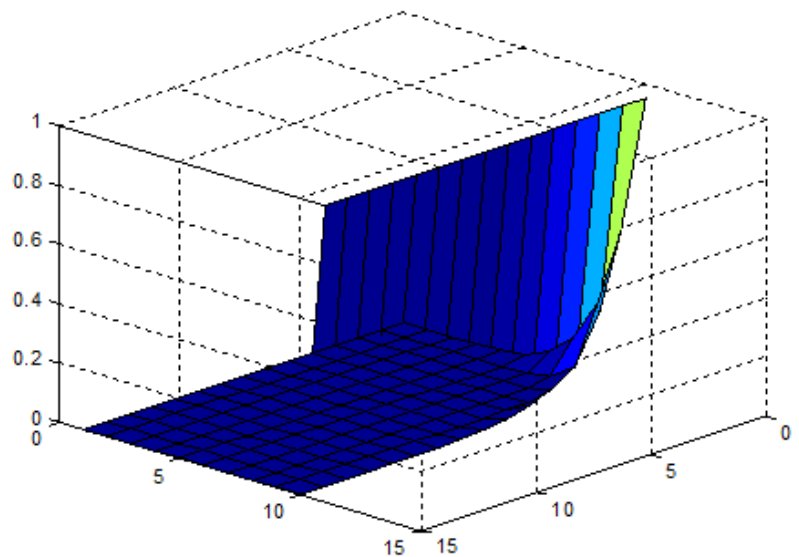

Fig. 6: Numerical solution

\section{Conclusion}

In this paper, we have applied the modified decomposition method for the solution of the heat equation with nonlocal boundary conditions. This algorithm is simple and easy to implement. The obtained results confirmed a good accuracy of the method. On the other hand, the calculations are simpler and faster than in traditional techniques 


\section{References}

[1]. G. Adomian,Nonlinear Stochastic Operator Equations, Academic Press, Florida, 1986

[2]. G. Adomian and R. Rach, On linear and nonlinear integro-differential equations, J. Math. Anal. Appl. 113 (1986), no. 1, $199-201$.

[3]. A. M. Wazwaz, A Reliable Modification of Adomian Decomposition Method, App. Math. And Computation, 102 (1999) 77-86.

[4]. A. M. Wazwaz, The modified decomposition method and Pade approximations for solving Thomas Fermi equations, App. Math. And Computation, 105 (1999) 11-19.

[5]. E. Babolian and S. H. Javadi, Restarted Adomian Method for Algebraic Equations, App. Math. And Computation, 146 (2003), 533541.

[6]. E. Babolian, S. H. Javadi, H. Sadeghi, Restarted Adomian Method for Integral Equations, App. Math. And Computation, 167 (2003), 1150-1155.

[7]. Hossein Jafari, Varsha Daftardar-Gejji, Revised Adomian decomposition method for Solving a system of nonlinear equations, App. Math. And Computation, 175 (2006), 1-7.

[8]. Hossein Jafari, Varsha Daftardar-Gejji, Revised Adomian decomposition method for Solving systems of Ordinary and Fractional Differential Equations, App. Math. And Computation, 181 (2006), 598-608.

[9]. A. Cheniguel and A. Ayadi," Solving Non Homogeneouse Heat Equation by the Adomian Decomposition Method ", International Mathematical Forum, Vol.6, 2011, no.13, 639-649

[10]. Momani S., dibat Z.O, "Analytical solutions of a time-fractional Navier-Stokes equation by Adomiande composition method", Appl. Math. Comput.Vol. 177, 2006, No.2, pp. 488-494.

[11]. Wazwaz A., "A reliable modification of Adomian decomposition method", Appl. Math. Comput., 102 (1), 1999 , pp. 77-86. 